\title{
CUSTO HORÁRIO DE UM TRATOR AGRÍCOLA ALIMENTADO COM DIFERENTES PROPORÇÕES DE ÓLEO DIESEL E BIODIESEL DE PINHÃO MANSO
}

Wagner da Cunha Siqueira ${ }^{1}$, Haroldo Carlos Fernandes ${ }^{2}$, Fabio Lucio Santos ${ }^{3}$, Anderson Candido da Silva ${ }^{4}$, Selma

Alves Abrahão ${ }^{5}$

\section{RESUMO}

Vários estudos estão sendo realizados utilizando o conjunto trator/equipamento para avaliar o seu desempenho em conjunto com combustível convencional (óleo Diesel). Dada à necessidade de novas alternativas para essa fonte finita de energia, os testes com combustíveis alternativos tornaram-se muito importantes. Portanto, objetivou-se com este trabalho analisar o custo horário de um trator agrícola, utilizando combustíveis com diferentes proporções de óleo Diesel (OD) com biodiesel de pinhão manso (OPM). Verificou-se, por meio de ensaios dinamométricos, o consumo específico de combustível do motor alimentado com diferentes misturas de combustíveis. Posteriormente, determinou-se o custo horário do trator utilizando o consumo obtido nos ensaios dinamométricos para as misturas de OD com OPM. O menor consumo específico de combustível foi verificado para o óleo Diesel. A mudança de combustível provocou variação no consumo específico do motor, sendo que o biodiesel de pinhão manso apresentou o maior consumo específico em todas as cargas aplicadas. Os menores valores de consumo específico foram observados quando são aplicadas cargas de 778 e 1023 N. A utilização de misturas de óleo Diesel com biodiesel de pinhão manso se apresentou economicamente viável.

Palavras-chave: consumo específico, ensaio dinamométrico, óleo vegetal

\section{ABSTRACT \\ HOURLY COST OF A FARM TRACTOR FUELED WITH DIFFERENT PROPORTIONS OF DIESEL AND JATROPHA OILS}

Studies are being conducted using the tractor / equipment to evaluate their performance in conjunction with conventional fuel (diesel oil). Given the need for new alternatives to this finite energy source, testing with alternative fuels has become very important. Therefore, this study sought to analyze the hourly cost of an agricultural tractor, using fuel blends of diesel oil (OD) with jatropha oil (OPM). Testing with a dynamometer indicated the specific fuel consumption of the engine fed with blends of OD and OPM. Thereafter, the hourly cost of the tractor was determined using consumption obtained by the dynamometer for blends of OD with OPM. Lower specific fuel consumption was recorded for Diesel oil. The change of fuel caused a variation in the specific consumption of the engine, and the jatropha biodiesel showed the highest specific consumption in all applied loads. The lowest values of specific fuel consumption were observed when loads of 778 and $1023 \mathrm{~N}$ are applied. Use of mixtures of diesel oil with jatropha biodiesel showed to be economically viable.

Keywords: dyno testing, vegetable oil, specific consumption

Recebido para publicação em 18/10/2013. Aprovado em 24/03/2014.

1 - Eng. Agrícola, Professor - IFMT, Cáceres - MT, wagner.cunha@cas.ifmt.edu.br

2 - Eng. Agrícola, Professor Associado, DEA/UFV, Viçosa - MG, haroldo@ufv.br

3 - Eng. Agrícola, Professor Adjunto, DEA/UFV, Viçosa - MG, fabio.ls@ufv.br

4 - Eng. Agrônomo, Mestrando Eng ${ }^{\mathrm{a}}$ Agrícola, DEA/UFV, Viçosa - MG, andersoncandido@hotmail.com

5 - Eng. Agrimensor, Profa. - IFMT, Cáceres - MT, selma.abrahao@cas.ifmt.edu.br 


\section{INTRODUÇÃO}

A utilização de óleos vegetais in natura, ou em mistura com óleo Diesel, para alimentação de motores de combustão interna pode ser viável, tomando-se alguns cuidados e fazendo algumas adaptações no motor para melhorar a injeção do combustível. As sementes do pinhão manso produzem um óleo de qualidade superior ao óleo de mamona e semelhante ao óleo Diesel, possibilitando seu uso como combustível alternativo ao óleo oriundo do petróleo em motores de ciclo Diesel (BANAPURMATH et al., 2008; SIRISOMBOONA et al., 2007).

Os estudos de comportamento de motores operando com biodiesel não detectaram significativos desgastes e redução da durabilidade, entretanto, observa-se que o aumento da proporção do biodiesel em mistura com óleo Diesel implica em maiores valores de consumo de combustível devido ao menor poder calorífico do biodiesel (TABILE et al., 2009).

Ao estudar o desempenho de um trator agrícola alimentado com biodiesel destilado, proveniente de óleo de frituras, Soranso et al. (2008), constataram aumento no consumo específico quando é utilizada maior proporção de biodiesel na mistura de combustíveis, sendo que o aumento médio no consumo específico para o combustível com $100 \%$ de biodiesel em relação ao óleo Diesel foi de 18\%.

A adoção de novos combustíveis para abastecimento das máquinas agrícolas deve ser amparada pelo estudo de custos relativos à sua utilização. $\mathrm{O}$ estudo do custo operacional de máquinas agrícolas deve ser realizado com base nos custos fixos que são debitados independentemente da utilização da máquina, estando incluída a depreciação, juros, alojamentos e seguros e dos custos operacionais, que dependem da quantidade do uso que se faz da máquina, estando incluídos os custos com combustível, manutenção, salários e lubrificantes (INOUE, 2008). Portanto, objetivouse com o presente trabalho realizar um estudo dos custos da utilização de misturas de óleo Diesel (OD) com biodiesel de Pinhão Manso (OPM) em um trator agrícola.

\section{MATERIAL E MÉTODOS}

O presente trabalho foi realizado no Laboratório de Mecanização Agrícola (LMA), pertencente ao Departamento de Engenharia Agrícola (DEA), da Universidade Federal de Viçosa (UFV). Foi avaliado o desempenho de um trator agrícola de pneus, marca Valmet, modelo 65ID, com aproximadamente 3000 horas de trabalho, tomada de potência tipo 1 em bom estado de conservação, alimentado com óleo Diesel, biodiesel de pinhão manso e três misturas com diferentes proporções entre esses dois combustíveis, contendo 25,50 e $75 \%$ de óleo Diesel. Após a avaliação, foi realizada a análise do custo horário do trator utilizando as cinco variações de mistura. As misturas foram separadas com base em peso e não em volume, devido à diferença de massa específica entre o $\mathrm{OD}$ e o OPM.

A tomada de potência do trator avaliado foi acoplada, por meio de um eixo cardã ao dinamômetro de rotação do tipo transmissão montado em berço. Além do dinamômetro, foi utilizado um fluxômetro de combustível com capacidade para $1.000 \mathrm{~mL}$, um tacômetro digital e de contato, cronômetro digital e uma célula de carga com capacidade máxima de $25 \mathrm{kN}$, marca Alfa Instrumentos ${ }^{\circledR}$ acoplada ao dinamômetro. As avaliações seguiram a norma NBR 5484 (1985) e para isto foram utilizadas temperaturas de bulbo úmido, bulbo seco e dados da pressão atmosférica, obtidos junto ao Instituto Nacional de Meteorologia (INMET).

O experimento foi conduzido no esquema de parcelas subdivididas segundo o delineamento inteiramente casualisado (DIC) para o ensaio na TDP, com sete cargas $(\mathrm{C} 1, \mathrm{C} 2, \mathrm{C} 3, \mathrm{C} . . ., \mathrm{C} 7)$ variando de 44,5 a 1.289,9 N (10 a $290 \mathrm{lbf})$, que não foram sorteadas e sim aplicadas de forma crescente conforme a norma NBR 5484 (1985), cinco combustíveis (M1, M2,M..., M5) e cinco repetições. Sendo assim, o fator mistura foi designado às parcelas e o fator cargas foi designado às subparcelas do experimento. Os dados foram submetidos à análise de variância e aplicação de teste de Tukey em cada carga aplicada para identificar a mistura que proporciona os melhores resultados nas variáveis de interesse.

$\mathrm{O}$ ensaio iniciou-se após cinco minutos de funcionamento do motor com óleo Diesel puro para aquecimento. Para os combustíveis com OPM, 
seguiu-se por mais cinco minutos de funcionamento do motor com a mistura a ser testada sob uma determinada carga. Para o encerramento, deixouse também o motor funcionando novamente cinco minutos com óleo Diesel puro, a fim de evitar formação de gomas e entupimentos.

$\mathrm{Na}$ avaliação foram coletados dados de rotação do motor, carga aplicada pelo dinamômetro e consumo de combustível para obtenção dos valores de consumo horário e consumo específico de combustível em função da rotação do motor.

Para cada carga aplicada pelo dinamômetro, esperou-se dois minutos para a estabilização da rotação, após esta estabilização verificou-se o nível de combustível no fluxômetro e a partir desta verificação a avaliação se processou por três minutos de funcionamento do trator. Durante esse intervalo de tempo foram coletados os dados citados anteriormente.

Os dados foram tabulados com o auxílio de planilha eletrônica, convertidos e corrigidos de acordo com a norma NBR 5484 (1985), utilizouse uma planilha para ensaio na tomada de potência que plota automaticamente as curvas características da potência, torque e consumo específico de combustível (FERNANDES, 2003).

Os componentes do custo foram estimados pela metodologia proposta pela Embrapa (PACHECO, 2000), conforme a Equação 1.

$\mathrm{CT}=\mathrm{CF}+\mathrm{CV}$

em que,

$\mathrm{CT}=$ Custo Total;

$\mathrm{CF}=$ Custos fixos; e

$\mathrm{CV}=$ Custos variáveis.

Os custos fixos são aqueles que devem ser debitados, independentemente da máquina ser usada ou não, daí o fato de também serem chamados de custos de propriedade. Entre os custos fixos são incluídos: depreciação (D), juros (J), alojamento e seguros (AS).

A depreciação se refere à desvalorização da máquina em função do tempo, seja ela utilizada ou não. Neste trabalho foi utilizado o método da linha reta e o valor de aquisição utilizado foi o valor correspondente ao modelo e ao ano de fabricação do trator.
A depreciação é estimada conforme Equação 2:

$D=\frac{V i-s}{V u}$

em que,

$\mathrm{D}=$ Depreciação $\left(\mathrm{R} \$ \mathrm{~h}^{-1}\right)$;

$\mathrm{Vi}=$ Preço de aquisição da máquina $(\mathrm{R} \$) ;$

$\mathrm{S}=$ Valor de sucata $=0,1 \times \mathrm{Vi}(\mathrm{R} \$)$; e

$\mathrm{Vu}=$ Vida útil (horas).

Os juros foram calculador conforme a Equação 3:

$J=\frac{\frac{[V i+(0,1 . V i)] i}{2}}{1000}$

em que,

$\mathrm{J}=\operatorname{Juros}\left(\mathrm{R} \$ \mathrm{~h}^{-1}\right)$;

$\mathrm{Vi}=$ Preço de aquisição $(\mathrm{R} \$)$;

$\mathrm{i}=$ Juros ao ano (decimal); e

1000 Horas trabalhadas no ano.

Segundo Pacheco (2000), os valores sugeridos para alojamento e seguro de máquinas variam de $0,75 \%$ a $1 \%$ do custo inicial ao ano para cada um desses dois componentes do custo fixo. Sendo assim, aconselha-se uma taxa de $2 \%$ ao ano para o cálculo conjunto do custo com alojamento e seguro, conforme Equação 4:

$A S=\frac{0,02 \mathrm{Vi}}{t}$

em que,

$\mathrm{AS}=$ Alojamento mais seguro $\left(\mathrm{R} \$ \mathrm{~h}^{-1}\right)$;

$\mathrm{Vi}=$ Preço de aquisição (R\$); e

$\mathrm{t}=$ Tempo de uso (horas ano ${ }^{-1}$ )

Os custos variáveis ou operacionais são aqueles que dependem da quantidade de uso que se faz da máquina e são constituídos por: combustíveis (C), lubrificantes (L), reparos e manutenção (RM) e salário do tratorista (ST) conforme Equação 5. 
Quadro 1. Estimativa do custo em litros para cada mistura avaliada

\begin{tabular}{|c|c|c|c|c|c|c|c|c|c|c|}
\hline \multicolumn{2}{|c|}{ Mistura } & $\%$ & $\begin{array}{c}\text { Massa esp } \\
(\mathrm{g} / \mathrm{ml})\end{array}$ & $\mathrm{g} \mathrm{L}^{-1}$ & $\begin{array}{r}\mathrm{g} \mathrm{Kg}^{-1} \text { de } \\
\text { Mistura }\end{array}$ & $\begin{array}{c}\text { mL em } 1 \\
\text { Kg }\end{array}$ & $\begin{array}{c}\mathrm{L} \text { em } 1 \\
\mathrm{Kg}\end{array}$ & $\begin{array}{c}\text { OPM } \\
(0,55 \text { R\$) }\end{array}$ & $\begin{array}{c}\text { OD } \\
(2,05 \mathrm{R} \$)\end{array}$ & $\begin{array}{l}\text { Custo / } \\
\text { L de } \\
\text { Mistura }\end{array}$ \\
\hline \multirow{2}{*}{1} & OPM & 0 & 0,7806 & 780,6 & 0 & 0 & 0 & 0 & - & \multirow{2}{*}{2,63} \\
\hline & OD & 100 & 0,7806 & 780,6 & 1000 & 1281,066 & 1,281 & - & 2,63 & \\
\hline \multirow{2}{*}{2} & OPM & 25 & 0,7957 & 795,7 & 250 & 314,189 & 0,314 & 0,17 & - & \multirow{2}{*}{2,14} \\
\hline & OD & 75 & 0,7806 & 780,6 & 750 & 960,799 & 0,961 & - & 1,97 & \\
\hline \multirow{2}{*}{3} & OPM & 50 & 0,8063 & 806,3 & 500 & 620,117 & 0,620 & 0,34 & - & \multirow{2}{*}{1,65} \\
\hline & OD & 50 & 0,7806 & 780,6 & 500 & 640,533 & 0,641 & - & 1,31 & \\
\hline \multirow{2}{*}{4} & OPM & 75 & 0,8063 & 806,3 & 750 & 930,175 & 0,930 & 0,51 & - & \multirow{2}{*}{1,17} \\
\hline & OD & 25 & 0,7806 & 780,6 & 250 & 320,266 & 0,320 & - & 0,66 & \\
\hline 5 & OPM & 100 & 0,8276 & 827,6 & 1000 & 1208,313 & 1,208 & 0,66 & - & 0,66 \\
\hline
\end{tabular}

$\mathrm{CV}=\mathrm{C}+\mathrm{L}+\mathrm{RM}+\mathrm{ST}$

É difícil avaliar com precisão o consumo de combustível dos tratores, devido às condições variáveis de carga a que são submetidos durante os trabalhos de campo. Os cálculos relativos ao custo com combustível foram realizados utilizando o preço de produção do biodiesel pinhão manso. A estimativa do custo de cada mistura esta representada no Quadro 1.

A quantidade de lubrificantes consumidos por hora depende do tipo e da potência do trator e pode ser obtida no manual do proprietário e na planilha de manutenção proposta pelo fabricante, determinando a capacidade dos reservatórios de lubrificantes e a periodicidade em horas em que devem ser substituídos. Para análise do custo de lubrificantes, foi utilizado o valor de $2 \%$ com relação ao consumo de cada carga aplicada ao trator.

Dentre as despesas de manutenção, que devem ser computadas para o cálculo do custo de operação de máquinas agrícolas, encontram-se aquelas realizadas para a manutenção preventiva e corretiva. Estima-se um valor igual a $100 \%$ do investimento inicial, durante toda a vida útil da máquina.

Para se calcular o custo horário da mão de obra foi utilizada a Equação 6, que leva em consideração benefícios e encargos sociais, além do salário base do operador. Foram considerados apenas os dias úteis durante o mês, ou seja, um número de 176 $\mathrm{h}$ trabalhadas por mês. O salário do tratorista foi considerado de dois salários mínimos por mês.

$S O=\frac{S+E}{D \times H}$

em que,

$\mathrm{SO}=$ Salário do operador, $\left(\mathrm{R} \$ \mathrm{~h}^{-1}\right)$;

$\mathrm{S}=2 \times$ Salário mínimo, $\left(\mathrm{R} \$\right.$ mês $\left.^{-1}\right)$;

$\mathrm{E}=$ Encargos sociais (considera - se $70 \%$ sobre o salário do operador)

$\mathrm{D}$ = Número de dias úteis trabalhados no mês, $(22$ dias mês $\left.{ }^{-1}\right)$; e

$\mathrm{H}=$ Número de horas trabalhadas por dia (8 horas $\left.\operatorname{dia}^{-1}\right)$.

\section{RESULTADOS E DISCUSSÃO}

No quadro 2 e Figura 1 estão representados, respectivamente, os dados e as curvas de consumo específico de combustível, corrigidos para as diferentes misturas avaliadas. A mudança de mistura provocou variação no consumo específico

\section{REVENG


de combustível, sendo que o biodiesel de pinhão manso apresentou o maior consumo específico em todas as cargas analisadas.

Nas cargas de 44,4 N e $289 \mathrm{~N}$, o consumo específico de combustível foi estatisticamente menor para a mistura com $25 \%$ de óleo Diesel, seguido pelo Óleo Diesel e aumentando nas outras misturas de acordo com o acréscimo de OPM quando utilizada a carga de 44,4 N. Esses resultados assemelham-se aos encontrados por Lopes (2006) onde o autor atribui esse comportamento ao menor poder calorífico do biodiesel.
Nas cargas de 533 N, 1023 N e 1267 N, o óleo Diesel e a mistura com $75 \%$ de óleo Diesel não diferiram estatisticamente entre si e apresentaram menor consumo específico de combustível em relação às misturas com maior proporção de biodiesel. Ao estudar o desempenho de um motor ciclo diesel alimentado com diferentes proporções de óleo Diesel e óleo de soja Castellanelli et al. (2008), relatam que o consumo específico do biodiesel apresenta valores mais próximos ao do óleo Diesel quando o motor trabalha em rotações mais elevadas.

Quadro 2. Médias do consumo específico $\left(\mathrm{g} \mathrm{kW}^{-1} \mathrm{~h}^{-1}\right)$ para os diferentes combustíveis

\begin{tabular}{cccccccc}
\hline \multicolumn{7}{c}{ Cargas (N) } \\
\hline Combustível & 44,4 & 289 & 533 & 778 & 1023 & 1267 & 1289 \\
\hline Óleo Diesel & $1673,35 \mathrm{~d}$ & $417,01 \mathrm{c}$ & $316,67 \mathrm{~b}$ & $278,95 \mathrm{~d}$ & $277,11 \mathrm{c}$ & $315,56 \mathrm{c}$ & $384,64 \mathrm{~b}$ \\
$75 \%$ OD & $1589,44 \mathrm{e}$ & $368,23 \mathrm{~d}$ & $315,37 \mathrm{~b}$ & $281,83 \mathrm{~cd}$ & $283,41 \mathrm{c}$ & $328,32 \mathrm{c}$ & $367,48 \mathrm{c}$ \\
$50 \%$ OD & $1816,01 \mathrm{c}$ & $437,35 \mathrm{~b}$ & $319,52 \mathrm{~b}$ & $304,65 \mathrm{~b}$ & $306,91 \mathrm{~b}$ & $351,84 \mathrm{~b}$ & $396,11 \mathrm{~b}$ \\
$25 \%$ OD & $1849,12 \mathrm{~b}$ & $413,19 \mathrm{c}$ & $320,94 \mathrm{~b}$ & $293,86 \mathrm{bc}$ & $301,36 \mathrm{~b}$ & $325,24 \mathrm{c}$ & $344,62 \mathrm{~d}$ \\
Biodiesel & $2971,40 \mathrm{a}$ & $663,81 \mathrm{a}$ & $510,14 \mathrm{a}$ & $490,43 \mathrm{a}$ & $470,43 \mathrm{a}$ & $525,14 \mathrm{a}$ & $561,56 \mathrm{a}$ \\
\hline
\end{tabular}

As médias seguidas por pelo menos uma mesma letra minúscula numa mesma coluna, não diferem estatisticamente pelo teste de Tukey, a 5\% de probabilidade.

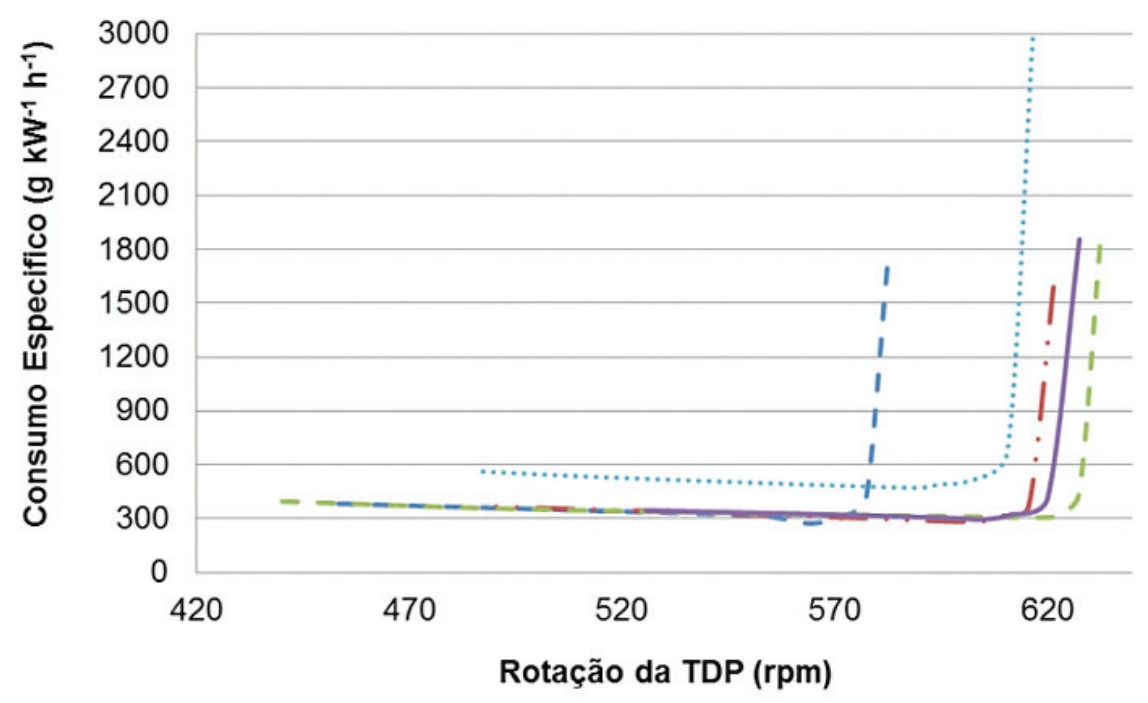

- $0 \%$ OPM - $25 \%$ OPM - - 50\%OPM - $75 \%$ OPM …... 100\%OPM

Figura 1. Curva do consumo específico de combustível para as diferentes misturas de combustível em função da rotação da TDP. 
Ao trabalhar com a carga de $533 \mathrm{~N}$, o óleo diesel e as misturas com 25, 50 e 75\% de OPM não diferiram entre si, porém apresentaram diferença em relação ao biodiesel de pinhão manso. Resultado semelhante foi observado por Vitoria et al. (2012) em que o menor consumo específico em todas as misturas de Óleo Diesel com Biodiesel de pinhão manso ocorreu em uma faixa de rotação entre 580 e 600 RPM, para uma carga aplicada a TDP de 888 N.

A operação com cargas mais elevadas apresenta os menores valores de consumo específico de combustível e diminui a diferença entre o consumo específico do óleo Diesel com as misturas contendo biodiesel, podendo tornar o custo para operação menor, devido ao baixo preço do biodiesel, mesmo que este apresente valores de consumo específico maiores que óleo Diesel. O custo operacional do trator utilizado foi estimado em $\mathrm{R} \$ \mathrm{~h}^{-1}$ para 1000 $\mathrm{h}$ trabalhadas por ano. Os valores utilizados para a estimativa dos custos podem ser visualizados no Quadro 3.

Conforme apresentado no Quadro 4, os custos estão apresentados com os valores acumulados ao final de cada ano trabalhado e por hora, de acordo com a vida útil estimada do trator.

Na composição dos custos variáveis, o custo dos combustíveis e a estimativa do óleo lubrificante, são os fatores que sofrem maior variação devido as diferentes proporções de OD e OPM. Os outros fatores como mão de obra e manutenção, não sofrem alteração com a mudança das cargas aplicadas. No quadro 5 é apresentada a estimativa do custo total em 5 anos ( vida útil) de cada mistura em cada carga aplicada pelo dinamômetro.

Observa-se mudança dos valores totais ao

Quadro 3. Valores utilizados para os cálculos do custo operacional

\begin{tabular}{|c|c|}
\hline Itens & Valores \\
\hline Valor de Aquisição (R\$) & $20.000,00$ \\
\hline Vida Útil ( Anos) & 5 \\
\hline Taxa de Juros (aa\%) & 10 \\
\hline Horas de Trabalho Anual & 1.000 \\
\hline Valor de Sucata (R\$) & 2.000 \\
\hline Óleo Lubrificante $\left(\mathrm{R} \$ \mathrm{~L}^{-1}\right)$ & 14,00 \\
\hline Custo do OD $\left(\mathrm{R} \$ \mathrm{~L}^{-1}\right)$ & 2,05 \\
\hline Custo do OPM $\left(\mathrm{R} \$ \mathrm{~L}^{-1}\right)$ & 0,55 \\
\hline \multicolumn{2}{|c|}{ Custo por Litro de Mistura (R\$) } \\
\hline OD & 2,63 \\
\hline $75 \%$ OD & 2,14 \\
\hline $50 \% \mathrm{OD}$ & 1,65 \\
\hline $25 \% \mathrm{OD}$ & 1,17 \\
\hline OPM & 0,66 \\
\hline
\end{tabular}

\section{REVENG}


Quadro 4. Custo fixo operacional

\begin{tabular}{cccccc}
\hline \multicolumn{7}{c}{ Custos Fixos } \\
\hline Vida Util (ano) & 1 & 2 & 3 & 4 & 5 \\
Depreciação $\left(\mathrm{R} \$\right.$ Ano $\left.^{-1}\right)$ & 3600 & 3600 & 3600 & 3600 & 3600 \\
Juros $\left(\mathrm{R} \$ \mathrm{Ano}^{-1}\right)$ & 1100 & 1100 & 1100 & 1100 & 1100 \\
ISA $\left(\mathrm{R} \$ \mathrm{Ano}^{-1}\right)$ & 400 & 400 & 400 & 400 & 400 \\
Total $\left(\mathrm{R} \$ \mathrm{~h}^{-1}\right)$ & 5,10 & 5,10 & 5,10 & 5,10 & 5,10 \\
\hline Total $\left(\mathbf{R} \$\right.$ Ano $\left.^{-1}\right)$ & $\mathbf{5 1 0 0}$ & $\mathbf{5 1 0 0}$ & $\mathbf{5 1 0 0}$ & $\mathbf{5 1 0 0}$ & $\mathbf{5 1 0 0}$ \\
\hline
\end{tabular}

Quadro 5. Custo total em 5 anos para cada mistura e para cada carga aplicada.

\begin{tabular}{|c|c|c|c|c|c|c|c|}
\hline \multicolumn{8}{|c|}{ Custo total para a mistura com $100 \%$ OD e $0 \%$ OPM em 5 anos de vida Útil } \\
\hline Cargas $(\mathrm{N})$ & 44,4 & 289 & 533 & 778 & 1023 & 1267 & 1289 \\
\hline Total $\left(\mathrm{R} \$ \mathrm{~h}^{-1}\right)$ & 28,06 & 31,44 & 34,93 & 38,54 & 43,48 & 51,92 & 52,21 \\
\hline Total $(\mathrm{R} \$)$ & 140297 & 157175 & 174635 & 192677 & 217412 & 259607 & 261062 \\
\hline \multicolumn{8}{|c|}{ Custo total (R\$) para a mistura com $75 \%$ OD e $25 \%$ OPM em 5 anos de vida Útil } \\
\hline Cargas $(\mathrm{N})$ & 44,4 & 289 & 533 & 778 & 1023 & 1267 & 1289 \\
\hline Total $\left(\mathrm{R} \$ \mathrm{~h}^{-1}\right)$ & 26,98 & 29,40 & 33,27 & 36,66 & 41,26 & 47,31 & 47,79 \\
\hline Total $(\mathrm{R} \$)$ & 134907 & 147007 & 166367 & 183307 & 206297 & 236547 & 238967 \\
\hline \multicolumn{8}{|c|}{ Custo total (R\$) para a mistura com 50\% OD e 50\% OPM em 5 anos de vida Útil } \\
\hline Cargas $(\mathrm{N})$ & 44,4 & 289 & 533 & 778 & 1023 & 1267 & 1289 \\
\hline Total $\left(\mathrm{R} \$ \mathrm{~h}^{-1}\right)$ & 26,60 & 29,00 & 31,31 & 34,98 & 39,03 & 41,85 & 42,04 \\
\hline Total $(\mathrm{R} \$)$ & 133015 & 144981 & 156561 & 174896 & 195161 & 209250 & 210215 \\
\hline \multicolumn{8}{|c|}{ Custo total (R\$) para a mistura com $25 \%$ OD e $75 \%$ OPM em 5 anos de vida Útil } \\
\hline Cargas $(\mathrm{N})$ & 44,4 & 289 & 533 & 778 & 1023 & 1267 & 1289 \\
\hline Total $\left(\mathrm{R} \$ \mathrm{~h}^{-1}\right)$ & 25,43 & 26,88 & 28,91 & 31,23 & 34,42 & 37,68 & 37,83 \\
\hline Total (R\$) & 127137 & 134387 & 144537 & 156137 & 172087 & 188400 & 189125 \\
\hline \multicolumn{8}{|c|}{ Custo total (R\$) para a mistura com $0 \%$ OD e 100\%OPM em 5 anos de vida Útil } \\
\hline Cargas (N) & 44,4 & 289 & 533 & 778 & 1023 & 1267 & 1289 \\
\hline $\operatorname{Total}\left(\mathrm{R} \$ \mathrm{~h}^{-1}\right)$ & 25,32 & 26,90 & 28,99 & 31,81 & 34,35 & 37,17 & 37,45 \\
\hline Total (R\$) & 126617 & 134513 & 144947 & 159047 & 171737 & 185837 & 187247 \\
\hline
\end{tabular}

longo da vida útil do trator conforme a alteração das misturas e das cargas aplicada. O quadro 6 apresenta a porcentagem da variação dos valores em relação ao custo quando é utilizado o óleo
Diesel como combustível.

O aumento da carga aplicada e da concentração de OPM nos combustíveis implica no aumento dos valores de diferença em relação ao óleo Diesel, 
Quadro 6. Porcentagem da variação dos valores em relação ao custo quando se utiliza óleo Diesel como combustível

Custo total (R\$) para o combustível com $100 \%$ OD e $0 \%$ OPM em 5 anos de vida Útil

\begin{tabular}{cccccccc}
\hline Cargas $(\mathrm{N})$ & 44,4 & 289 & 533 & 778 & 1023 & 1267 & 1289 \\
\hline Total & 140297 & 157175 & 174635 & 192677 & 217412 & 259607 & 261062 \\
\hline
\end{tabular}

Diferença porcentual dos valores utilizando misturas de OPM em relação ao uso de $100 \% \mathrm{OD}$

\begin{tabular}{cccccccc}
\hline Misturas/Cargas (N) & 44,4 & 289 & 533 & 778 & 1023 & 1267 & 1289 \\
\hline $75 \%$ OD e $25 \% \mathrm{OPM}$ & 3,84 & 6,47 & 4,73 & 4,86 & 5,11 & 8,88 & 8,46 \\
\hline $50 \%$ OD e $50 \% \mathrm{OPM}$ & 5,19 & 7,76 & 10,35 & 9,23 & 10,23 & 19,40 & 19,48 \\
\hline $25 \%$ OD e $75 \% \mathrm{OPM}$ & 9,38 & 14,50 & 17,23 & 18,96 & 20,85 & 27,43 & 27,56 \\
\hline $0 \%$ OD e $100 \% \mathrm{OPM}$ & 9,75 & 14,42 & 17,00 & 17,45 & 21,01 & 28,42 & 28,27 \\
\hline
\end{tabular}

Diferença dos valores (R\$) correspondente a cada porcentagem das misturas de OPM em relação ao uso de $100 \%$ OD

\begin{tabular}{cccccccc}
\hline Misturas/Cargas (N) & 44,4 & 289 & 533 & 778 & 1023 & 1267 & 1289 \\
\hline $75 \%$ OD e 25\%OPM & 5390,00 & 10168,00 & 8268,00 & 9370,00 & 11115,00 & 23060,00 & 22095,00 \\
\hline $50 \%$ OD e 50\%OPM & 7282,00 & 12194,00 & 18074,00 & 17781,00 & 22251,00 & 50357,00 & 50847,00 \\
\hline $25 \%$ OD e $75 \%$ OPM & 13160,00 & 22788,00 & 30098,00 & 36540,00 & 45325,00 & 71207,00 & 71937,00 \\
\hline $0 \%$ OD e 100\%OPM & 13680,00 & 22662,00 & 29688,00 & 33630,00 & 45675,00 & 73770,00 & 73815,00 \\
\hline
\end{tabular}

demonstrando uma economia, no custo em 5 anos da vida útil do trator, podendo variar de 5.390,00 a 73.815,00 reais. A partir da carga de $289 \mathrm{~N}$ e do uso do combustível com $25 \%$ de OD, a economia proporcionada representa, aproximadamente, o valor de aquisição do trator em estudo.

\section{CONCLUSÕES}

- O consumo específico de combustível para o biodiesel é maior que todos os outros combustíveis independentemente da carga aplicada;

- Em todas as misturas de combustíveis avaliados os menores valores de consumo específico são obtidos quando se utiliza as cargas de 778 e $1023 \mathrm{~N}$;

- A utilização de qualquer mistura entre óleo
Diesel e biodiesel de pinhão manso se apresenta economicamente viável, em relação ao óleo Diesel, independentemente da carga aplicada.

\section{REFERÊNCIAS BIBLIOGRÁFICAS}

ASSOCIAÇÃO BRASILEIRA DE NORMAS TÉCNICAS. NBR 5484: Motores alternativos de combustão interna de ignição por compressão (Diesel) ou ignição por centelha (Otto) de velocidade angular variável - ensaio - Método de ensaio. Rio de Janeiro, 1985. 8p.

BANAPURMATH, N.R.; TEWARI, P.G.; HOSMATH, R.S. Performance and emission characteristics of a DI compression ignition engine operated on Honge, Jatropha and sesame oil methyl esters. Renewable Energy, v.33,n.9, p.1982-1988, 2008.

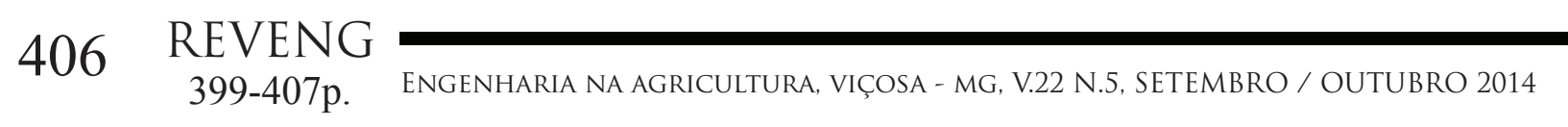


CASTELLANELLI, M.; SOUZA, S.N.M.; SILVA, S.L.; KAILER, E.K. Desempenho de motor ciclo diesel em bancada dinamométrica utilizando misturas diesel/biodiesel. Engenharia Agrícola, Jaboticabal, v.28, n.1, p.45-153, 2008.

INOUE, G.H. Utilização de óleo vegetal em motor estacionário de ciclo diesel. 2008. 102f. Tese (Doutorado em Engenharia Agrícola). Universidade Federal de Viçosa, Viçosa, 2008.

LOPES, A. Biodiesel em trator agrícola: desempenho e opacidade. 2006. 158f. Tese (Livre - Docência em Agronomia) - Faculdade de Ciências Agrárias e Veterinárias de Jaboticabal, Universidade Estadual Paulista, Jaboticabal, 2006.

PACHECO, E.P. Seleção e custo operacional de máquinas agrícolas. Rio Branco: Embrapa Acre, 2000. 21p. (Embrapa Acre. Documentos, 58).

SIRISOMBOONA, P.; KITCHAIYAB, P.; PHOLPHOA, T.; MAHUTTANYAVANITCHA, W. Physical and mechanical properties of Jatropha curcas L. fruits, nuts and kernels. Biosystems Engineering, v.97, p.201-207, 2007.
SORANSO, A. M.; GABRIEL FILHO, A.; LOPES, A.; SOUZA, E.G.; DABDOB, M.J.; FURLANI, C.E.A.; CAMARA, F.T. Desempenho dinâmico de um trator agrícola utilizando biodiesel destilado de óleo residual. Revista Brasileira de Engenharia Agrícola e Ambiental, v.12, p.553-559, 2008.

TABILE, R.A.; LOPES, A.; DABDOUB, M.J.; CAMARA, F.T.; FURLANI, C.E.A.; SILVA, R.P. Biodiesel de mamona no diesel interior e metropolitano em trator agrícola. Eng. Agríc., Jaboticabal, v.29, n.3, Sept. 2009 . Available from $<$ http://www.scielo.br/scielo.php?scri p t $=\mathrm{s} \mathrm{ci}_{-}$a r t t e x t \& p i d = S $0100-$ $69162009000300008 \& \operatorname{lng}=\mathrm{e} \& n r m=i s o>$. access on 20 Mar. 2014. http://dx.doi.org/10.1590/ S0100-69162009000300008.

VITÓRIA, E.L; OLIVEIRA, P.S; FERNANDES, H.C; LONGUI, F.C; LEITE, D.M. Desempenho comparativo de um motor ciclo Diesel alimentado com misturas de óleo Diesel e biodiesel de pinhãomanso. Enciclopédia biosfera, Centro Científico Conhecer - Goiânia, v.8, n.14, p.1865, 2012. 